\title{
Why observational studies are important in comparative effectiveness research: the effect of breast-conserving therapy and mastectomy in the real world
}

\author{
Marissa C. van Maaren ${ }^{1,2}$, Philip Poortmans ${ }^{3}$, Luc J. A. Strobbe ${ }^{4}$, Linda de Munck ${ }^{1}$, Sabine Siesling ${ }^{1,2}$ \\ ${ }^{1}$ Department of Research, Netherlands Comprehensive Cancer Organisation, Utrecht, the Netherlands; ${ }^{2}$ Department of Health Technology \\ \& Services Research, MIRA Institute for Biomedical Technology and Technical Medicine, University of Twente, Enschede, the Netherlands; \\ ${ }^{3}$ Department of Radiation Oncology, Radboud University Medical Center, Nijmegen, the Netherlands; ${ }^{4}$ Department of Surgical Oncology, Canisius \\ Wilhelmina Hospital, Nijmegen, the Netherlands \\ Correspondence to: Marissa C. van Maaren. Department of Research, Netherlands Comprehensive Cancer Organisation, P.O. Box 19079, 3501 DB \\ Utrecht, the Netherlands. Email: m.vanmaaren@iknl.nl. \\ Provenance: This is an invited Correspondence commissioned by Section Editor San-Gang Wu (Department of Radiation Oncology, Xiamen Cancer \\ Center, the First Affiliated Hospital of Xiamen University, Xiamen, China). \\ Response to: Franceschini G, Orlandi A, Sanchez AM, et al. Mastectomy in precision oncology era: myth or reality? Transl Cancer Res 2016;5:S544-5.
}

Submitted Nov 15, 2016. Accepted for publication Nov 23, 2016.

doi: $10.21037 /$ tcr.2016.12.53

View this article at: http://dx.doi.org/10.21037/tcr.2016.12.53

We thank Dr. Franceschini and colleagues for their comment (1) on our recently published article in the Lancet Oncology (2). First, it is completely justified to ask oneself whether results from observational studies are likely to arise from selection bias. It is extensively described that observational studies investigating treatment effects are prone to confounding by severity (3). However, in contrast to randomised controlled trials (RCTs), observational studies do reflect the real-world population. As long as we interpret the results carefully, as it should be done for RCTs as well, these types of studies are of additional value through their ability to include large numbers of patients in conditions as we see them in daily practice $(4,5)$. Hereby, results from observational studies generate valuable information that can support findings obtained from RCTs. Especially regarding the 'battle' between breast-conserving surgery plus radiotherapy (BCT) and mastectomy (MAST), all RCTs where clinicians rely on, were conducted in the nineteen-eighties. Over the last decades, diagnostic procedures, (adjuvant) treatment and follow-up strategies have been improved drastically. Besides, the proportion of elderly breast cancer patients is increasing. This may all contribute to altered survival rates in the present era and motivated us to investigate primary treatment effects on a population-based observational cohort as seen in daily practice.

To elaborate on the possibly large impact of age on the outcomes, we would like to mention that we used age as a categorical variable in the multivariable analyses. We categorised patients into six groups: $<40,40-49,50-59$, $60-69,70-79,>79$ years, as shown in our baseline table (2). Indeed, patients in the MAST group (median age 63 years) were generally older than patients in the BCT group (median age 58 years). Although we corrected for age in the multivariable analyses, it may still have an impact on the outcome. Future research should define whether the results hold in every age group.

Another example the authors mention is stratification for biological prognostic factors such as oestrogen receptor (ER) and progesterone receptor (PR) status. We completely agree that these factors may play an important role in survival outcomes. As discussed in our article, HER2 status testing was in our cohort not routinely introduced in clinical practice yet, as it wasn't either in the era of the RCTs. Therefore, it was not used in clinical decisionmaking. Lacking data on HER2 status certainly limits the generalisability of the results to the current setting, as mentioned in our discussion section, especially for the relatively small subgroup of patients with HER2 overexpression. The authors rightly note that ER and 
PR receptor status were missing for a large proportion of patients. Although missing data was considered as missing at random, this is certainly a limitation of our study. However, to prevent exclusion of a large number of patients, we performed multiple imputation. This statistical method is described to lead to the generation of unbiased, but more precise effect estimates as compared with complete case analyses (6). In this way, we were able to correct for ER and PR receptor status without excluding patients, still obtaining reliable estimates. To further confirm if the results hold true in subgroups based on molecular subtype, stratified analyses must be performed in a more recent cohort, in which ER, PR and HER2 status are routinely determined. The authors also mention the Ki67 receptor as important biological prognostic factor. We would like to emphasise that Ki67 is a controversially discussed parameter in studying breast cancer prognosis, of which standardised assays should be developed to facilitate comparisons between studies (7). Moreover, a proliferation factor (mitotic count) is included in the Bloom-Richardson grading of breast cancer, as practised in the Netherlands.

The authors conclude that the above-described factors have led to an imbalance of the treatment groups, resulting in improved survival for BCT compared to MAST in T1N0 breast cancer. We agree with the authors that a certain imbalance is present. As we also describe in our discussion section, we cannot completely eliminate confounding by severity and residual confounding in observational studies (2). Therefore, we interpret the results with care, stating that BCT is at least equivalent to MAST. These results are a confirmation of the fact that BCT performs at least equal in terms of overall and breast cancer-specific survival in early breast cancer in the real-world population. Taking into account the cosmetic and psychological aspects, we hope to reduce the number of patients that choose for MAST based on unjustified fear of recurrent cancer and death. MAST still remains a viable option in T1N0 breast cancer, especially since there are many other factors playing a role in the decision-making for BCT or MAST, of which the patient's wish should not be forgotten.

\section{Acknowledgements}

None.

\section{Footnote}

Conflicts of Interest: The authors have no conflicts of interest to declare.

\section{References}

1. Franceschini G, Orlandi A, Sanchez AM, et al. Mastectomy in precision oncology era: myth or reality? Transl Cancer Res 2016;5:S544-5.

2. van Maaren MC, de Munck L, de Bock GH, et al. 10 year survival after breast-conserving surgery plus radiotherapy compared with mastectomy in early breast cancer in the Netherlands: a population-based study. Lancet Oncol 2016;17:1158-70.

3. Barton $\mathrm{S}$. Which clinical studies provide the best evidence? The best RCT still trumps the best observational study. BMJ 2000;321:255-6.

4. Hershman DL, Wright JD. Comparative effectiveness research in oncology methodology: observational data. J Clin Oncol 2012;30:4215-22.

5. Giordano SH. Comparative effectiveness research in cancer with observational data. Am Soc Clin Oncol Educ Book 2015:e330-5.

6. Janssen KJ, Donders AR, Harrell FE Jr, et al. Missing covariate data in medical research: to impute is better than to ignore. J Clin Epidemiol 2010;63:721-7.

7. Luporsi E, André F, Spyratos F, et al. Ki-67: level of evidence and methodological considerations for its role in the clinical management of breast cancer: analytical and critical review. Breast Cancer Res Treat 2012;132:895-915.
Cite this article as: van Maaren MC, Poortmans P, Strobbe LJ, de Munck L, Siesling S. Why observational studies are important in comparative effectiveness research: the effect of breast-conserving therapy and mastectomy in the real world. Transl Cancer Res 2016;5(Suppl 7):S1549-S1550. doi: 10.21037/tcr.2016.12.53 